\section{P-51 PROVIDING HOSPICE CARE AT HOME FOR PEOPLE WHO DON'T LIVE IN A TRADITIONAL 'HOME' OR WHO ARE HOMELESS}

Warren Finney. Prospect Hospice, Swindon, UK

10.1136/bmispcare-2017-hospice.78

Last year Prospect Hospice supported around 450 people with life-limiting illnesses at home through our Hospice at Home service, but what if you don't live in a traditional 'home'? How do you access end-of-life care in the place you call home? We recognise that home can mean a shared supported house for people who are vulnerable, a care home, a hostel, a $\mathrm{B}$ and $\mathrm{B}$, or even a local park. This is where the people most important to them are, where they have lived and felt safe for many years. Our priority is to ensure excellent end-of-life care for everyone, no matter where home is.

We have improved care for the most vulnerable in their place of choice by:

- Identifying people that do not have access to traditional Hospice at Home services. Initially this has included people living in care homes, people living in supported housing with learning disabilities and people who are homeless.

- Working with staff at these organisations to ensure everyone can access the care they need in the place they call 'home'

- Identifying barriers blocking the delivery of care.

- Designing training (some with formal NCFC qualifications) enhancing the knowledge and skills of staff to support their clients' needs.

- Creating recognised links to the hospice to improve on-going care.

- Securing local recognition of the lack of end-of-life care for people who are homeless

- Improving patient care across all three client groups by supporting 33 care homes or other organisations and training 214 of their staff.

- Increasing referrals as a result of our training for people who homeless and for dedicated CNS care home referrals from people living shared living accommodation.

- Improving confidence of frontline staff in delivering care for residents in the setting of their choice through support and training. Our care home support has just won a national Charity Civil Society Award for Healthcare.

\section{\begin{tabular}{|l|l}
\hline P-52 & BRIDGING THE GAP BETWEEN LEARNING DISABILITIES \\
\hline
\end{tabular} AND PALLIATIVE CARE}

${ }^{1}$ Helen Miller, ${ }^{2}$ Louise Jenkins. ${ }^{1}$ Isabel Hospice, Welwyn Garden City, UK; ${ }^{2}$ Adult Disability Service, Farnham House, Stevenage, UK

10.1136/bmjspcare-2017-hospice.79

Background People with learning disabilities (PLD) are approximately $2 \%$ of the UK population (Tuffrey-Wijne et al., 2016). Improved health and social care means people live longer, resulting in increased need for palliative care services for all. Current research demonstrates multiple inequalities in palliative care for PLD (Heslop et al., 2013; Tuffrey-Wijne \& McLaughlin, 2015). As an experienced Clinical Nurse Specialist in palliative care, whilst reflecting on the needs of PLD, I found knowledge gaps in the hospice workforce and complexity about end-of-life care delivery. Working with learning disability health professionals, there was little knowledge of palliative care services and poor understanding of collaborative support and co-ordination at end of life.

Aim To reduce the knowledge gap of hospice and community nursing staff with reference to palliative care for PLD and facilitate co-ordination and collaboration.

Method Delivered an educational day called 'Bridging the Gap between Learning Disabilities and Palliative Care' for hospice and community staff working with PLD. Material used was adapted from the East of England $\mathrm{ABC}$ programme and national learning disabilities documents (National End of Life Care Programme, 2011). Advertised through a hospice education programme and supported by a learning-disabilities lead nurse.

The Day Covered Definitions of learning disabilities and inequalities. Communication skills, Advance Care Planning and awareness of easy-read documentation. Complex drug management and symptom control at end of life, including epilepsy and enteral feeding. Collaboration and co-ordination with local programmes to engage hospices in becoming learning-disability-friendly organisations (Hertfordshire County Council, 2013).

Results

- 14 people attended from the community and three different hospices.

- Average satisfaction score for the day was 9.5 out of 10 .

- Confidence levels, from 1 Low to 5 high, were measured against seven objectives, pre- and post- education. Average confidence rose from 3 pre to 4.7 post.

- All hospices are keen to become learning-disabilities-friendly organisations.

Conclusion There are clear gaps in knowledge of both learning disability and hospice staff. Education is vital to filling these gaps and hospices are key to facilitating co-ordinated and collaborative care.

\section{P-53 WORKING TOGETHER TO IMPROVE ACCESS FOR PATIENTS WITH A LEARNING DISABILITY}

Ruth Isherwood, Hazel Gaylor. Strathcarron Hospice, Denny, UK

10.1136/bmjspcare-2017-hospice.80

Background The palliative care needs of people with a learning disability have been recognised (The keys to life strategy recommendations, 2013; Redall, 2010; Tuffrey-Wijne, 2003). There is a need for accessible information and joint working by learning disability and palliative care teams. There may be particular challenges around assessment of capacity, communication, delayed diagnosis and thorough assessment of symptoms.

Aim The project describes the service currently provided by specialist palliative care to patients with a learning disability in order that we can continue to improve access and service for this group of patients.

Method Following a significant event analysis involving the two teams, a retrospective case note review was undertaken to identify themes which would help inform further service development. All patients with a learning disability who were 